\title{
THE REVERSED MOSCOW METHOD. A GENERAL FRAMEWORK FOR DEVELOPING AGE-FRIENDLY TECHNOLOGIES
}

\author{
Luiza Spiru ${ }^{1}$, Mircea Mărzan ${ }^{1}$, Cosmina Paul $^{1}$, Magdalena Velciu ${ }^{1}$ and Adrian Garleanu ${ }^{2}$ \\ ${ }^{1}$ Ana Aslan International Foundation \\ Spătarului Street, no 3, Bucharest, Romania \\ ${ }^{2}$ Independent Researcher
}

\begin{abstract}
In the last decade, the number of new technologies which target independent and active seniors at home tremendously increased, though many failed to address seniors' expectations and needs. The purpose of this study was to construct and test a new framework for developing age-friendly technologies for independent and active seniors at home. The proposed framework is the 'Reversed MoSCoW Method', where MoSCoW stands for must, should, could and have requirements to accomplish business needs. The tool is a prioritization for reaching a common ground among the technology developers and designers on not to do requirements, when conceptualizing new technologies for independent and active seniors at home. The proposed model was grounded on the research findings from Senior-TV. Hence, a questionnaire survey was conducted with a sample of 148 seniors aged 65 and over in Cyprus, Romania and Slovenia, between February and April 2019, and the results are transferred and tested in a current ongoing European AAL project, IOANNA. The results showed that understanding the specificities of the relationship between technology and the independent and active seniors is crucial for understanding their habits and dispositions towards adopting new technologies. The findings show the most frequent mistakes when developing new technologies for active seniors: 1) age stigmatization, 2) the tendency to improve already domesticated products or services, such as TV watching or communication by phone, as seniors do not engage on Internet at the expense of TV watching or on social media instead of communication by phone, 3) asking for a continue or frequent logging in, 4) health oriented services, 5) services' complexity and others. These were found as better predictors of failing technologies than the conventionally assumed attitudinal factors, such as ease of use, disinterest or lacking technological abilities.
\end{abstract}

\section{KEYWORDS}

Age-Friendly Technology, Independent Seniors, “Reversed Moscow Method', Gerontographics

\section{INTRODUCTION}

Senior population is ageing and its diversity is increasing (European Comission, 2018). Independent and active seniors at home are seen both as a demographic resource and as a societal concern for ageing well and so technologies are called to play a crucial role to ease the role of independent and active seniors in societies and communities, and to keep them ageing well. Even the paradigmatic understanding of aging as a natural process is challenged today, as traditionally ageing is associated with a reduced mobility, chronic diseases and mental health disorders. Gerontologists propose to look at the ageing process rather as a disease and the focus to be put on improving the health and wellbeing of the senior population (Bulterijs et al, 2015). Technologies of the near future for healthy aging are fast developing and they are rapidly moving into the home (National Research Council, 2011). New age-friendly technologies offer innovative solution for helping people to stay as healthy and independent as possible. The challenge is to develop the right technologies such as elderly would benefit greatly from products, and designing is crucial. Age-friendly technologies are used to promote healthy living, preserve physical and mental capacity and delaying normal brain aging (Spiru L., 2011), also enable seniors to continue to live well. 
In order to develop an age-friendly technology to promote innovation and healthcare solutions targeted to the elderly, it is important to have a proper understanding of the elderly user requirements and their significance. An in-depth understanding of the elderly as a distinct group in all respects, such as the way of living (independent or not), status of health, physical and psychological support (physical, emotional, cognitive), socio-economical aspects, their relation with technology is key for a correct approach into the near future of technologies for ageing well. Willing to offer elders all they need, some projects are based on incorrect assumption and requirements and find later that the elderly requests are others that they believed.

We bring together our research findings from two projects part of Active Assisted Living (AAL) Programme, which aimed at promoting information technology for aging well and deliver healthy ageing solutions to market. By using these transferable results from various field-researchers conducted over the last decade, we want to illuminate the reality of interests, needs and habits of seniors related to technology use. The promoted AAL projects are: 1) IOANNA, which will develop six services: stores update tool, end-user interaction tool, route planner, optimum product or service choice, user-friendly interface and a mobile application [IOANNA, 2019], and 2) SENIOR-TV, which aimed at developing a platform for providing formal and informal caregiving services to older adults that live alone in their own homes, at low cost, and that focuses on the active prevention and the maintenance of relationships with friends, family, and the community [Senior-TV, 2019]

The paper's contribution resides on creating a framework based on the research findings and lessons learned from previous projects on the needs of independent and active seniors, when designing technologies which target them. There are necessary steps before putting down the elderly user requirements meaning what the elderly users expect the product or technology to do. Choosing the right requirements is essential for the success of the product, and avoid the failure. Especially in the case of elderly, the prioritization of the needs is as important as the prioritizations of refrains and anxieties, in order to reach technological adoption benefits. The process of prioritizing is an activity for defining what requirements to achieve or not to do, and in what sequence. It is a method to make the project more efficient by identifying the most wanted characteristics to offer.

\section{BODY OF PAPER}

We need to go behind the trite division of young and old seniors or other groupings based on age, as it has been shown that the heterogeneity of the senior population is much higher than that of other demographic groups (Nimrod, 2013; Sthienrapapayut et al, 2018). There are various types of segmentation of the elderly market which have been employed by economics and marketing when aiming at an understanding of this mature market. Though, due to the large heterogeneity of the people aged over 65 , segmentations such as sociodemographic, behavioral or psychographic proved insufficiently explanatory (Nimrod, 2013, Moschis, 1996).

According to the French Silver Economy strategy, launched by the Ministère des Affaires Sociales et de la Santé in December 2013, the aging population is defined in 3 groups; active, fragile and dependent. Moschis developed the gerontographics approach in order to assess the high variability of the seniors' needs. The proposed gerontographics segmentation of the adult market is developed from the individual differences in aging, and the variances in the life experiences which influence psychographics factors. Four segments of the elderly are identified based on the type of aging experienced: healthy indulgers, ailing outgoers, healthy hermits and frail recluses (Moschis and Mathur 1993; Moschis 1996, 2003). One group is covering the segmentation of independent seniors living at home, and they are healthy indulgers. Healthy indulgers are seniors who experienced less negative life events. Comparative to others, they have a good health condition which supports their psychological and social aging. They continue to age well, staying independent, active, and socially integrated, enjoying life and sharing similar behavior with those younger. The other groups manifest either a psychological or a physical health decline, which either insulate them from society or develop various grades of dependency from their informal or formal caregivers.

The projects who fall under the scope of this article selected persons according to age criterion and included people soon-to-be seniors, aged 55 years and over, from three ranges: 55-65; 65-75 and 75+ years, with balanced distribution. According to health status, the target group is formed by healthy persons who are active or persons with some (minor) problems of health, independent or with physical disability. 


\subsection{The Relationship of Independent Seniors at Home with Technologies}

The field research conducted during the project Senior-TV in Cyprus, Romania and Slovenia and which interviewed 57 persons, from a total sample of 148 which included also other categories of seniors based on their self-perceived health status. They were identified as healthy indulgers, namely independent and socially active seniors living at home, and two relevant findings concerning their relation with technology: 1) that their predisposition for using technologies decline with time, and 2) that is merely due to the perceived irrelevance of technology in their life.

Table 1. Independent seniors living at home and their relation with technology

\begin{tabular}{|c|c|c|c|c|}
\hline & \multirow{2}{*}{ Enter houses } & \multirow{2}{*}{$\begin{array}{c}\text { Average } \\
\text { First encounter } \\
\quad \text { (years) }\end{array}$} & \multicolumn{2}{|c|}{ USAGE } \\
\hline & & & Property & Frequency of usage on average \\
\hline Radio & 1940 & - & Rather not & Few times a week \\
\hline TV & 1960 & 20 & ALL HAVE & Daily \\
\hline Landline Phone & 1980 & - & Rather Not & Few times a week \\
\hline Mobile phone & \multirow{4}{*}{2000} & 59 & WIDESPREAD & Few times a week \\
\hline Computer & & 60 & \multirow{2}{*}{ Either have or don't have } & Few times a week \\
\hline Internet & & 62 & & Few times a week \\
\hline E-mail & & & Rather not & Occasionally \\
\hline Smart phones & 2010 & & Either have or don't have & Either daily or never \\
\hline Smart TV & 2015 & & Rather not & Either daily or never \\
\hline Smart Watch & 2015 & & Inconclusive & - \\
\hline
\end{tabular}

Senior-TV project findings show that healthy indulgers are those who encounter various technologies earlier in life, comparative to the other three categories, though their anxiety towards technology increase in time and so does the perceived irrelevance of technology in their life. Healthy hermits keep a constant anxiety towards technology, and frail recluses' anxiety towards new technology decreases with time.

It is the 'perceived usefulness' of technology which matters for independents seniors at home and encourage them towards exploring and experimenting new technologies, comparative to other seniors who are embolden by the influences of their informal and formal caregivers and social entourage.

When developing new technologies, the ranking on seniors' future services preferences should be taken into account. According to the research conducted over the Senior-TV project, from February to Aprilie 2019 in Romania, Cyprus and Slovenia, the first concerns of the elderly refer to their safety, independency and socializing. Their top three technologies preferred are: 1) the sensors which warn about a tap or cooker left open; stove and oven safety control; 2) Sensors for preventing or detecting falls and 3) Organizing walking groups. Seniors from Cyprus and Slovenia add as one of their first interests also the 'online websites for seniors' services'. Therefore, the extent to which new technologies meet seniors' concerns should be of primary interest for scholars and practitioners from the conceptualization phase and not only over the developmental process.

\subsubsection{The Reversed MoSCoW Method for Developing Technologies for Independent Seniors at Home}

We have developed a 'Reversed MoSCoW Method' to serve as a general framework for guiding technology developers and designers for new technologies, which target independent seniors living at home. This framework is generally addressed for people over 65 years old but the self-perception of age, cultural and environmental settings also accounts for the adaptability of this framework. 
MoSCoW approach refers to specific user requirements that depend on the development of a specific product or service targeting independent seniors living at home, specifically, to the must, should, could and would or won't of a product for market success. The prioritization of these requirements can be conducted according to MoSCow method. Because we discuss here universal requirements of the seniors, we have built requirements which are embedded in a "not to" framework, which we called "The Reversed-MoSCoW Method" and are equally relevant to technology development processes.

"Must NOT have" refers to critical requirements. "Should NOT have" are the important requirements though not critical, and for the case of elderly, are often as important as "Must NOT have" because social influences and societal environmental factors are much weaker than for the other categories of age, such as people on the job or youngsters. The difference consist in the fact that seniors would accept or adopt a technology which meet "the Must NOT" requirements but they will not continue to use it, or it will not became a natural habit for them if the "Should not have" requirements are not met. "Could NOT have" are desirable but not necessary, and could improve user experience or satisfaction. The least relevant requirements are listed at the end, in the "Won't have" category.

Must NOT have. Age-stigma: new technologies need to be promoted as universal technologies and not as gerontechnologies, meaning that when marketing new technologies to independent seniors it is important to address universality and not social stigma of ageing. These findings are in line with Moschis (2003) research findings on advertising universality. Echo the domesticated technologies: new technologies do not need to tend to either replace or duplicate already existent information, communication \& entertainment services. For example, the following needs cannot be replaced by new technology devices because of the domestication process which they went through: the use of TV for information and phone for communication. Family and friends and television are mentioned as the first two most important sources of enjoyment and, moreover, studies indicate that the time spent on Internet and face to face interactions do not tend to replace TV watching (Östlund, 2010). Continues or frequent logged in: Participants refer to their usage of mobile technology as an activity that is performed 'only when it is necessary'. Participants explicitly state that they do not wish to be constantly connected; main reasons are privacy issues and the time needed to learn to use complex mobile devices. Additionally, they need to 'save' their sensory and cognitive resources for other things that require attention while on the way. Technology developers should also consider that independent seniors are not particularly interested in social networks (Senior-TV and T\&T projects).

Should NOT have: Health oriented services: many times, services developed for independent elderly at home concern emergencies, supervision, monitoring health or help which are rather meant for assisted elderly and should be used by their carers. Independent and active seniors manifest interest in enjoyment and information and not in health preventive measures. Independent seniors living at home tend to appeal to professional medicals for their health management and not to new technologies, as they do not want to constantly be reminded of their age and possible frailty condition. This finding has its cultural limitation and should be tested against seniors with various educational and cultural backgrounds. Development process without seniors' involvement: Conceptualization, development and design conducted by middle-age people without the involvement of the elderly. Generally, the involvement of the seniors in the phase of conceptualization is ignored by the technology developers. Complexity (with a special emphasis on many choices): seniors dislike complexity and avoid the computer wherever possible. They keep a constant medium to high general anxiety towards technology, even though they used to integrate technology before in their social and working environments. Moreover, the participating seniors did not want many choices.

Could NOT have: Ignoring physical impairments. Research findings show that the 'Perceived ease of use' has an indirect impact on the behavioral usage of the independent seniors (Chen and Chan, 2013). Therefore, the ease of use considerations is integrated in 'could not have' category. Here, physical impairments should be factor in when developing and designing new technologies. Participants favor old remotes instead of the touch remote control, mention as desirable features big buttons, pleasant high-pitched voice, and lower complexity: few menu choices, low hierarchy of choices. The interface needs to be self-explicable, non-invasive and simple, perhaps at expense of other extra functionalities. Presumed experience in general technology abilities: the lack of experience of the independent seniors may be noticed in the small number of seniors who are familiar with GPS and many of them would not know how to connect to a Wi-Fi network. Moreover, a majority of seniors engage online only to do the necessary and do not check their accounts regularly. The previous AAL projects found that when participants plan a route, it is of equal relevance to them the user interface of the planning application and the output of this planning: the print, 
regular e-mail check, virtual contact instead of face to face contact. technological services without human contact as the trust decrease with age.

Would NOT be nice to have: intrusive features, money management and gaming. Most seniors are cautious of sharing personal data online, managing their bank accounts online and they refrain from playing games online, comparative to other groups of seniors who manifest more frailty and look for engaging online with games. Gaming online and on the phone is especially of interest to women who have a low psychological well-being, according to the Senior-TV research findings.

The below table present a systematic view of the above described research findings.
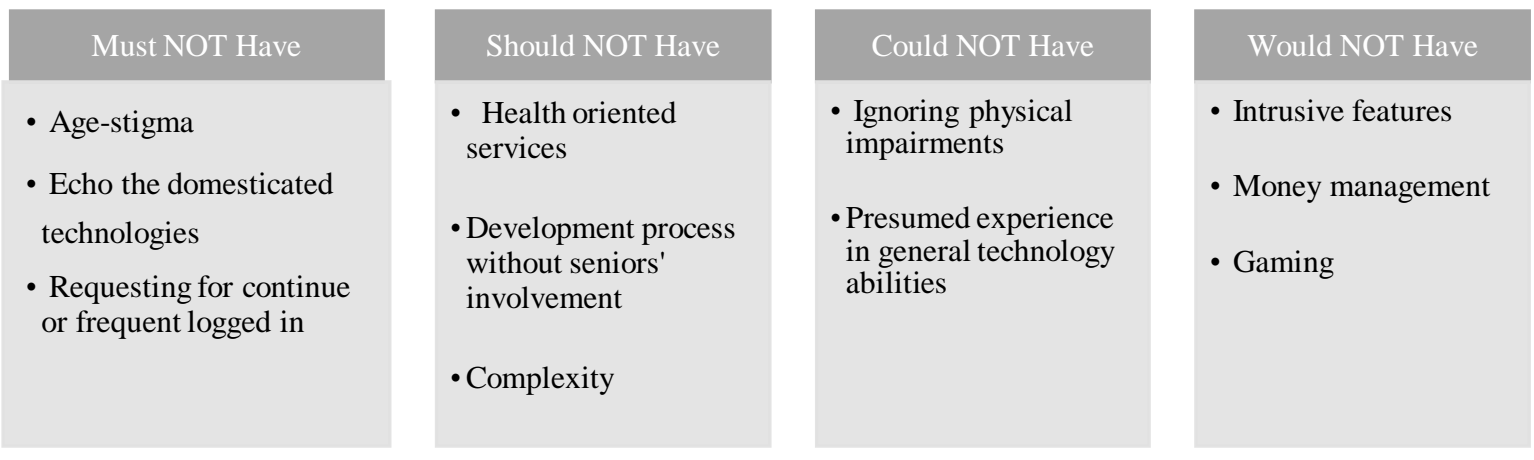

Figure 1. The Reversed MoSCoW Method. A general framework for developing technologies for independent and active seniors at home

\subsection{Using the Reversed MoSCoW Method to develop the integrated solution IOANNA}

The IOANNA application is an integrated solution that helps seniors for searching for commercial offers, services and specific products near the home (new technological features). The application is designed to make elderly feel safer, stay active, find a more convenient route and stay in contact with persons and healthcare service, in case of emergency. The solution also helps social engagement through community service in paid or volunteer basis for experienced senior adults and assistive mobility with the navigation system aiding senior adults or other citizens to get to their destination in the most convenient way. Design technology was seen as crucial as well as the age-friendly interface, with special care for seniors' needs. Research on users' requirements was focused on the assessment of the characteristics of real needs as well as identifying their expectations and preferences and even the unconscious determinants of making decisions process.

The field research conducted during the IOANNA project involves people identified as independent seniors living at home, and it shows that seniors' self-perceived health status and self-determining movement are very important, also keeping them connected with family, if needed. The evaluation was based on quantitative methods for analyzing perceptions and ideas in a measurable approach. The data were collected using in-depth interviews with senior adults and the elderly (over 55 years) in order to understand their needs, requirements, and ideas for each service of the IOANNA solution. Information is analyzed by themes for qualitative insights or through numerical comparisons for quantitative results. In September-October 2018, we interviewed 25 persons from Romania and 22 persons from Cyprus. Regarding IOANNA application, seniors perceive that the idea of having all services in one product is very interesting and useful, and appreciate to have an easy to use application and save time. Independence and security are very important.

\subsubsection{Applying the Reversed MoSCow Method for Elderly-User Requirements}

After collecting the users' requirements, we employed the 'Reversed MoSCoW Method' to test the degree to which the product answer to the specified not requirements model. The MoSCoW method is a technique used in the development of new technology to identify the most important requirements to deliver and so, the involvement of targeted people is mandatory. 
Must not have requirements have been met to a medium degree. The IOANNA application avoid age-stigma by developing an innovative advanced solution on mobile smart-phone in order to help people to stay active and by addressing elderly with respect. The findings show that the seniors' preference for using Internet on a personal computer and smartphone rather than a tablet may lead 1) to the perception that the already existing online information is duplicated and 2) to make seniors to prefer to connect on computer which is offering them the possibility of printing, which is shown as being highly appreciated by seniors, instead of using a permanent online connection, which refrains seniors from using a service.

Should not have requirements are met to a high degree. The IOANNA project followed the user needs analysis that was conducted in previous successfully Independent and seniors' involvement is a reliable mean to ensure that requirements and needs of elders are investigated and respected in every stage of the project in order to create a product which is useful, easy to use, with high acceptability. If addressing complexity, the project is meeting various expectations that decrease the acceptance of seniors who rather prefer simple and focused services.

Could not have requirements are met to a medium degree. IOANNA application is mobile which may affect the adoption of the application because of physical impairments. The fact that seniors prefer to have their app with them in order to be able to use the notification function if something happens to them implies the appealing to the service for emergency, which just a side feature. To permanently stay connected for checking the route planning is a presumption of experience in general technology abilities, which should not have been made.

Would not be nice to have requirements are met to a medium extent. Our results show that seniors order travel, transport ticket more frequently and less frequently for health issues, maybe because of the lack of trust. Tailoring IOANNA application that offers a verified network with a more secured link and limited access of unknown persons is just a presumed and not tested solution for countering seniors' lack of trust.

\section{CONCLUSION}

The present developed framework, 'the Reversed MoSCoW Method' refers to the prioritization of the 'Not to do requirements', when developing new technologies which target independent and socially active seniors at home. The here proposed method is meant to serve scholars and practitioners to deepen their understanding about developing technologies which potentate and maintain the well ageing of the independent and active seniors and their involvement in the society. Despite this positive contribution, there are two major limitations to the extant research. We discuss here just the universal requirements for the independent and active seniors at home, which are not transferrable to seniors who do not live independently and are insulated from society because of physical or psychological ill-being and who manifest a certain degree of dependency. Another limitation is that this framework does not account for culturally diverse factors and it might be relevant only to the Eastern and Southern Europe, as the Western Europeans seniors may exhibit a different relationship with new technologies. However, the wide application of the framework in new product development is of great relevance for supporting the decision process even from its very incipient phases.

\section{ACKNOWLEDGEMENT}

This work was performed in the frame of the EU project IOANNA (AAL-2017-077, with implementation period April 2018 - Sept 2020, funded by the AAL Programme, co-funded by the European Commission and the National Funding Authorities of Cyprus, Spain and Romania) and The Senior-TV project (AAL-2014-171, that is co funded by the EU AAL Joint programme and the related national Authorities in Spain, The Netherlands, Cyprus, Romania and Slovenia. 


\section{REFERENCES}

Bulterijs, S. at al, 2015, It is time to classify biological aging as a disease. Frontiers in genetics, vol. 6, no. 205.

European Commission, 2017, The 2018 Ageing Report, Luxembourg: Publications Office of the European Union.

Ioanna AAL Projects. http://www.aal-europe.eu/projects/ioanna/ [Online]. [Accessed from: 25 May, 2019].

Ministère des Affaires Sociales et de la Santé, December 2013. Silver Economy Strategy. [Online] http://www.socialsante.gouv.fr/espaces,770/personnes-agees-autonomie,776/dossiers,758/silver-economie,2432/. [Accessed from: 29 May, 2019].

Moschis, G.P, 2003. Marketing to older adults: an updated overview of present knowledge and practice. Journal of Consumer Marketing, vol. 20 no 6, pp.516-525.

Moschis, G.P, 1996. Gerontographics: Life-stage segmentation for marketing strategy development, Greenwood Publishing Group.

Moschis, G.P. et al, 1997. Targeting the mature market: opportunities and challenges. Journal of consumer marketing, vol. 14 no 4, pp.282-293.

Moschis, G.P., Mathur A, 1993. How they're acting their age. Marketing Management, vol. 2 no 2, p.40.

National Research Council, 2011, Health Care Comes Home: The Human Factors. Washington, DC: The National Academies Press, pp https://doi.org/10.17226/13149.

Nimrod, G., 2013, Probing the Audience of Seniors' Online Communities, The Journals of Gerontology: Series B, Vol. 68, Issue 5, September 2013, pp 773-782, https://doi.org/10.1093/geronb/gbt059

Östlund, B., 2010. Watching television in later life: a deeper understanding of TV viewing in the homes of old people and in geriatric care contexts. Scandinavian journal of caring sciences, vol. 24 no 2, pp.233-243.

Senior-TV AAL Project. http://www.aal-europe.eu/projects/senior-tv/ [Online]. [Accessed from: 25 May, 2019]

Senior-TV Project. 2019. Deliverable 1.3 Part A "Analysis of existing services of formal and informal care".

Spiru L. and Turcu I, 2011, Nature and nurture: A golden concept for healthy brain aging, Alzheimer's \& Dementia: The Journal of the Alzheimer's Association, Volume 7, Issue 4, pp.

Sthienrapapayut Th. at al, 2018, "Using gerontographics to explain consumer behaviour in later life: Evidence from a Thai study", Journal of Consumer Marketing, vol. 35, issue 3, pp https://doi.org/10.1108/JCM-02-2017-2083 